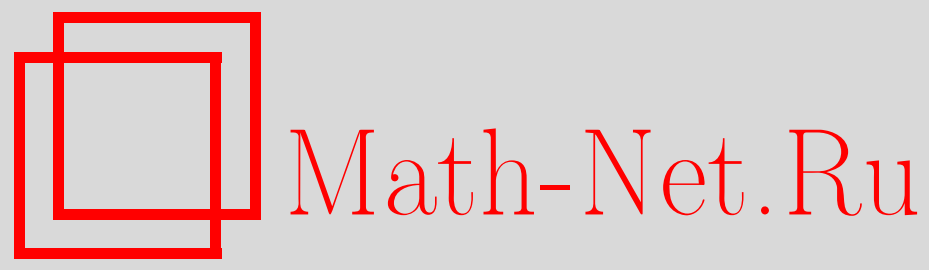

М. С. Агранович, Б. А. Амосов, Оценки $s$-чисел и спектральные асимптотики для интегральных операторов типа потенциала на негладких поверхностях, Функи. анализ и его прил., 1996, том 30, выпуск 2, 1-18

DOI: https://doi.org/10.4213/faa518

Использование Общероссийского математического портала Math$\mathrm{Net.Ru}$ подразумевает, что вы прочитали и согласны с пользовательским соглашением http://www . mathnet.ru/rus/agreement

Параметры загрузки:

IP : 54.166 .219 .16

26 апреля 2023 г., 05:18:43

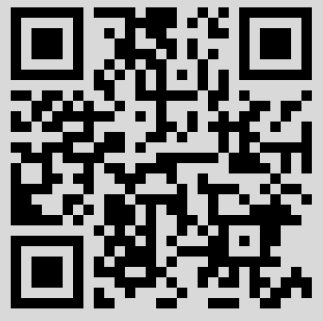


Функииональньй анализ и еәо приложения

1996, т. 30, вып. 2, с. 1-18

УДК 517.98

\title{
Оценки $s$-чисел и спектральные асимптотики для интегральных операторов типа потенциала на негладких поверхностях
}

\author{
(c) 1996. М. С. АГранович, Б. А. Амосов $^{1}$
}

\section{§1. Введение}

Пусть $S$ - замкнутая (т. е. компактная и без края) липшицева $(n-1)$-мерная поверхность в $\mathbb{R}^{n}$. Это означает, что локально, после подходящего поворота системы координат в $\mathbb{R}^{n}$, эта поверхность является графиком функции, удовлетворяющей условию Липшица:

$$
x_{n}=f\left(x^{\prime}\right) \quad\left(x^{\prime} \in \Omega \subset \mathbb{R}^{n-1}\right), \quad\left|f\left(y^{\prime}\right)-f\left(x^{\prime}\right)\right| \leqslant L\left|y^{\prime}-x^{\prime}\right|,
$$

где $\Omega$ - ограниченная область в $\mathbb{R}^{n-1}$. На $S$ естественным образом определяется «площадь» - $(n-1)$-мерная мера Лебега, индуцированная метрикой в $\mathbb{R}^{n}$. Предположим, что в $\mathbb{R}^{n}$ или в содержащем $S$ открытом множестве $\mathcal{O} \subset$ $\mathbb{R}^{n}$ задан полиоднородный, или классический, ПДО (псевдодифференциальный оператор) $\mathcal{A}$ порядка $m-1$, где $m<0$. Известно, что такой оператор является интегральным и его ядро $K(x, y)$ - бесконечно гладкая при $x \neq y$ функция, имеющая слабую особенность при $x=y$ с определенным асимптотическим разложением при $|x-y| \rightarrow 0$ (см. [1] или обзор [2], п. 1.6). Сужая эту функцию на $S \times S$, введем интегральный оператор

$$
A u(x)=\int_{S} \varphi(x) K(x, y) \psi(y) u(y) d S_{y} \quad(x \in S) .
$$

Здесь $d S$ - элемент площади на $S, \varphi(x)$ и $\psi(y)$ - измеримые функции на $S$ с модулем, не превосходящим единицы, например, индикаторы (характеристические функции) измеримых множеств на $S$. Отсутствие края у $S$ предполагается для упрощения изложения: наличие множителей $\varphi$ и $\psi$ по существу позволяет охватить случай, когда $S$ имеет край. В 33 эти множители будут использованы при выводе спектральных асимптотик.

Ядром оператора (1.2) является произведение $\varphi(x) K(x, y) \psi(y)$, но нам будет удобно функцию $K(x, y)$ тоже называть ядром. Это ядро имеет слабую особенность на $S$ (поскольку порядок оператора $\mathcal{A}$ не просто отрицателен, а меньше -1 ; см. [1] или [2]). Поэтому $A$ - компактный оператор в пространстве $L^{2}(S)$ со скалярным произведением $(u, v)=\int_{S} u \cdot \bar{v} d S$. Напомним, что $s$-числа $s_{j}(T)$ компактного оператора $T$ в гильбертовом пространстве $H$ - это положительные собственные значения неотрицательного оператора $\left(T T^{*}\right)^{1 / 2}$ (или

\footnotetext{
${ }^{1}$ Работа поддержана РФФИ, грант 95-01-00549, и ISF, грант N3H300.
} 
$\left(T^{*} T\right)^{1 / 2}$, где $T^{*}$ - оператор, сопряженный к $\left.T\right)$, занумерованные в порядке невозрастания с учетом кратностей.

Наша основная цель состоит в выводе оценки (напоминаем, что $m<0$ )

$$
\varlimsup_{j \rightarrow \infty} s_{j}(A) j^{-m /(n-1)} \leqslant C d(\varphi, \psi),
$$

где $C$ не зависит от $\varphi$ и $\psi$ (см. теорему 2.9 ). Величина $d(\varphi, \psi)$ определяется в п. 2.1а. Она выражается через $(n-1)$-мерные меры Лебега $m(\operatorname{supp} \varphi)$ и $m(\operatorname{supp} \psi)$ на $S$ и мала, если мала хотя бы одна из этих мер. Носитель функции определяется обычным образом - как замыкание множества точек, в которых она отлична от 0 . Точность показателя в (1.3) хорошо известна в случае гладкой поверхности $S$; см. также $\S 3$.

Основной пример - оператор (1.2) типа потенциала простого слоя для эллиптического дифференциального уравнения с бесконечно гладкими коэффициентами в $\mathcal{O}$; в этом случае $K(x, y)$ - фундаментальное решение такого уравнения. Фактически мы получим оценку (1.3) для более общих ядер, в частности, с конечной гладкостью: см. теорему 2.9 .

Наша вторая задача состоит в выводе асимптотической формулы для собственных значений оператора $A$ в более специальном случае, когда липшицева поверхность $S$ «почти гладкая» и $A$ - самосопряженный оператор в $L^{2}(S)($ см. теорему 3.2$)$. Мы называем замкнутую $(n-1)$-мерную липшицеву поверхность $S$ почти гладкой, если она имеет структуру открытого бесконечно гладкого $(n-1)$-мерного многообразия вне некоторого замкнутого «множества особенностей» $S_{0}$ с нулевой мерой Лебега $m\left(S_{0}\right)$ на $S$. В частности, почти гладкими являются поверхности конуса, цилиндра (например, с кругами в основаниях) и многогранника в $\mathbb{R}^{3}$, а также их образы при диффеоморфизмах. Конкретная геометрия такой поверхности не играет роли. Асимптотика имеет вид

$$
\lambda_{j}^{ \pm}(A)=b_{ \pm} j^{m /(n-1)}+o\left(j^{m /(n-1)}\right) \quad(j \rightarrow \infty)
$$

где $\lambda_{j}^{ \pm}(A)$ - положительные и отрицательные собственные значения оператора $A$, занумерованные с учетом кратностей в порядке невозрастания модулей; постоянные $b_{ \pm}$обычным образом вычисляются через главный символ ПДО $A$ на $S \backslash S_{0}$.

Для гладких поверхностей наши результаты близки к хорошо известным. Оценкам $s$-чисел и спектральным асимптотикам интегральных операторов посвящена обширная литература. Ближе всего к нашей работе цикл статей М. ШІ. Бирмана и М. 3. Соломяка, см. их обзор [3], в котором можно найти дальнейшие ссылки, и работы [4], а также работа Г. П. Костометова [5]. В них получены точные оценки $s$-чисел весьма общих интегральных операторов в областях $n$-мерного пространства и асимптотики собственных значений самосопряженных ПДО отрицательного порядка в таких областях.

Отметим также, что В. Я. Иврий рассмотрел в [6] самосопряженные ПДО отрицательного порядка на гладком замкнутом многообразии и получил для них асимптотики собственных значений с оценкой остатка и что А. А. Лаптев вывел в [7] из результатов, приведенных в [3], оценку $s$-чисел ПДО отрицательного порядка, действующего с $S_{1}$ в $S_{2}$, где $S_{1}$ и $S_{2}$ - гладкие $(n-1)$-мерные 
многообразия в $\mathbb{R}^{n}$ с пересечением $S_{1} \cap S_{2}$, имеющим нулевую $(n-1)$-мерную меру Лебега.

Нам понадобятся некоторые результаты и приемы из работ Бирмана-Соломяка. Основная трудность в рассматриваемой нами ситуации, в отличие от рассмотренных в этих работах, состоит в том, что сужение ядра $K(x, y)$ на $S \times S$ почти не сохраняет гладкости при $x \neq y$, существенной в подходе БирманаСоломяка в случае, когда особенность у ядра достаточно слабая (а именно, при $m \leqslant-(n-1) / 2)$. Можно было бы пытаться преодолеть эту трудность, аппроксимируя липшицеву поверхность гладкими поверхностями (такие аппроксимации описаны в $[8,9])$. Мы поступаем проще: аппроксимируем ядро $K(x, y)$ вырожденным ядром $K_{N}(x, y)$ вблизи $S \times S$, а затем сужаем на $S \times S$ оба ядра, $K$ и $K_{N}$. Как и в [3], $K_{N}$ строится при помощи кусочно-полиномиальных приближений к $K$; но у нас они строятся явно и очень просто: с использованием тейлоровских многочленов по $x$ или по $y$ для исходного ядра в одинаковых «малых кубах». Такой упрощенный подход пригоден и для вывода оценок типа (1.3) для ПДО отрицательного порядка в ограниченной области евклидова пространства или на гладкой поверхности без выхода в объемлющее пространство (см. замечание 2.10).

Конечно, для приложений было бы удобнее иметь оценки $s$-чисел в случае ядер, определенных только на $S \times S$, и для негладких $S$. В этом направлении мы получим теорему 4.1 для случая $-(n-1) / 2<m<0$. Она подсказана работой [5] об интегральных операторах в области пространства $\mathbb{R}^{n}$ (см. также ссылки в [5]). В доказательстве используется техника из [5] с некоторыми упрощениями. Независимо справедливость подобного результата заметил М. 3. Соломяк.

Возможные приложения наших результатов относятся к спектральным задачам для эллиптических уравнений и систем 2-го порядка в областях с негладкими границами со спектральным параметром в граничном условии. В настоящей работе мы ограничимся примерами таких задач для уравнения Лапласа в $\mathbb{R}^{3}$ (см. п. 4.1). Непростую аналитическую основу для этих рассмотрений составляют результаты работ по граничным задачам для гармонических функций и свойствам соответствующих поверхностных потенциалов в случае липшищевой границы. Мы сошлемся на работы Верхоты [9], Костабеля [10] и Джерисона-Кенига [11], в которых можно найти дальнейшие ссылки.

В этой связи отметим еще следующий случай, в котором можно несколько обобщить теорему 3.2. Предположим, что некоторый оператор $A$, действующий в $L^{2}(S)$, допускает два представления:

$$
A=T_{1} A_{1}=A_{2} T_{2},
$$

где $A_{1}$ и $A_{2}$ - операторы вида $(1.2)$, а $T_{1}$ и $T_{2}$ - ограниченные операторы в $L^{2}(S)$. Оценка $s$-чисел в этом случае тривиально получается из $(1.3)$ : например, $s_{j}(A) \leqslant\left\|T_{1}\right\| s_{j}\left(A_{1}\right)$. Но если $S$ - почти гладкая поверхность и $A-$ самосопряженный оператор в $L^{2}(S)$, сужение которого на гладкую часть $S \backslash S_{0}$ поверхности $S$ есть ПДО отрицательного порядка, то мы можем получить и асимптотику собственных значений для $A$ (см. п. 3.5). Такая ситуация возникает при рассмотрении «третьей краевой задачи» для эллиптических уравнений 2-го порядка со спектральным параметром в граничном условии (см. п. 4.1).

Авторы искренне благодарят М. 3. Соломяка за полезные обсуждения. 


\section{§2. Оценки $s$-чисел}

2.1. Нам понадобится следующая лемма о компактных операторах в гильбертовом пространстве, являющаяся простым следствием известной формулы

$$
s_{j+1}(A)=\min _{\operatorname{dim} B \leqslant j}\|A-B\|
$$

(см. [12, гл. II, п. 3]).

Лемма 2.1. Пусть $A_{N}(N=1,2, \ldots)-$ последовательность конечномерных операторов, такал, ито $\operatorname{dim} A_{N} \leqslant c_{1} N^{\nu} u\left\|A-A_{N}\right\| \leqslant c_{2} N^{-\alpha} n p u$ достаточно больших $N$, аде $c_{1}, c_{2}, \nu$ и $\alpha$ - положительные постолнные. Тогда

$$
\varlimsup_{j \rightarrow \infty} s_{j}(A) j^{\alpha / \nu} \leqslant c_{2} c_{1}^{\alpha / \nu} .
$$

ДокАЗАТЕЛЬСТво. Для достаточно большого $j$ найдется такое натуральное $N=N_{j}$, что $c_{1} N_{j}^{\nu}<j \leqslant c_{1}\left(N_{j}+1\right)^{\nu}$. Тогда в силу $(2.1)$

$$
s_{j+1}(A) \leqslant c_{2} N_{j}^{-\alpha} \leqslant c_{2}\left(\left(j / c_{1}\right)^{1 / \nu}-1\right)^{-\alpha},
$$

что и приводит к (2.2).

Для (1.2) подходящие $A_{N}$ мы определим явно в виде интегральных операторов

$$
A_{N} u(x)=\int_{S} \varphi(x) K_{N}(x, y) \psi(y) u(y) d S_{y} \quad(x \in S)
$$

с вырожденными ядрами $K_{N}$, которые сначала строятся в $\mathcal{O} \times \mathcal{O}$.

Условимся под нормой $\|w\|$ ядра $w(x, y)$ интегрального оператора

$$
W u(x)=\int_{S} w(x, y) u(y) d y
$$

действующего в $L^{2}(S)$, понимать норму $\|W\|$ этого оператора.

Для простоты обозначений мы всюду дальше будем предполагать, что $S$ содержится в единичном кубе $I^{n}=[0,1)^{n}$ (в квадрате при $\left.n=2\right)$. Рассмотрим равномерное разбиение этого куба в объединение $N^{n}$ попарно не пересекающихся «малых кубов» $V^{\alpha}=\left[\left(\alpha_{1}-1\right) h, \alpha_{1} h\right) \times \cdots \times\left[\left(\alpha_{N}-1\right) h, \alpha_{N} h\right)$ с ребром длины $h=1 / N$. Здесь $\alpha=\left(\alpha_{1}, \ldots, \alpha_{N}\right), \alpha_{i}=1, \ldots, N$. Нужные нам кусочнополиномиальные по $x$ аппроксимации $K_{N}$ ядра $K$ будут иметь вид

$$
K_{N}(x, y)=\sum_{|\beta| \leqslant r} k_{\alpha, \beta}(y) x^{\beta}, \quad x \in V^{\alpha}, y \in I^{n},
$$

где $\left.k_{\alpha, \beta}(y)\right|_{S} \in L^{2}(S)$. Очевидно, что операторы $A_{N}$ конечномерны.

2.1а. Пусть $q>-(n-1)$. Введем класс $\mathcal{K}^{q}$ определенных в $\mathcal{O} \times \mathcal{O}$ ядер $K(x, y)$ со следующими свойствами: 1$)$ существует последовательность $K_{N}(x, y)$ аппроксимаций вида (2.5) некоторой фиксированной степени $r$, такая, что на $S$

$$
\left\|K-K_{N}\right\|=O\left(N^{-q-n+1}\right) ;
$$

2) это же верно для ядра $\overline{K(y, x)}$. (Все функции пока считаются скалярными.) Ясно, что $\mathcal{K}^{q}$ - линеал и что при $q_{1}>q_{2}$ справедливо включение $\mathcal{K}^{q_{1}} \subset \mathcal{K}^{q_{2}}$. 
Для оценки размерности оператора (2.3) мы введем некоторые величины, связанные с подмножествами и функциями на $S$. Пусть сначала $X$ - любое множество на $S$. Обозначим через $\nu(X, N)$ число малых кубов $V^{\alpha}$ со стороной $1 / N$, имеющих непустое пересечение с $X$, и положим

$$
\mu(X)=\varlimsup_{N \rightarrow \infty} \frac{\nu(X, N)}{N^{n-1}} .
$$

Ясно, что $\mu(X) \leqslant \mu\left(X^{\prime}\right)$ при $X \subset X^{\prime}$; из приведенной ниже леммы 2.2 видно, что величина $\mu(S)$ конечна. Если $X=\operatorname{supp} \varphi$, то вместо $\nu(\operatorname{supp} \varphi, N)$ и $\mu(\operatorname{supp} \varphi)$ мы будем писать соответственно $\nu_{\varphi}(N)$ и $\mu_{\varphi}$.

ЛЕмма 2.2. Для замкнутых подмножеств $X \subset S$ величина $\mu(X)$ и мера Лебега $m(X)$ на $S$ эквивалентны, т.е.

$$
C_{1} \leqslant \mu(X) / m(X) \leqslant C_{2},
$$

где $C_{1}$ и $C_{2}$ - положительнье постояннье, не зависящие от $X$.

ДокАЗАтЕльство. 1) Пусть сначала $X$ - замкнутое множество, лежащее в $(n-1)$-мерном кубе $I^{n-1}=\left\{x \in I^{n}: x_{n}=0\right\}$. В этом случае $\nu(X, N) / N^{n-1}=$ $m\left(Q_{N}\right)$, где $Q_{N}$ - объединение малых $(n-1)$-мерных кубов $V^{\alpha^{\prime}}$ со стороной $1 / N$, имеющих обшие точки с $X$. Обозначим через $O_{\varepsilon}(X)$ открытую $\varepsilon$ окрестность множества $X$ на гиперплоскости $\mathbb{R}^{n-1}=\left\{x \in \mathbb{R}^{n}: x_{n}=0\right\}$ $(\varepsilon>0)$. Ясно, что $X \subset Q_{N} \subset O_{\sqrt{n} / N}(X)$; поэтому

$$
m(X) \leqslant m\left(Q_{N}\right) \leqslant m\left(O_{\sqrt{n} / N}(X)\right)
$$

Так как множество $X$ замкнуто, то оно совпадает с пересечением окрестностей $O_{\varepsilon}(X)$ по всем $\varepsilon>0$, и $m\left(O_{\varepsilon}(X)\right) \rightarrow m(X)$ при $\varepsilon \rightarrow 0$ в силу свойства непрерывности меры Лебега. Мы заключаем, что в данном случае $\mu(X)=m(X)$. Дальше в доказательстве мы ограничимся грубыми оценками.

2) Пусть теперь $X$ лежит на поверхности $\mathfrak{S} \subset I^{n}$, определяемой уравнением $x_{n}=f\left(x^{\prime}\right)$, где $f$ - липшицева функция. Эта функция почти всюду дифференцируема (см., например, $[13$, п. 3.1.6]), и мера $m(X)$ выражается интегралом

$$
m(X)=\int_{X_{0}} \sqrt{1+\left|\nabla f\left(x^{\prime}\right)\right|^{2}} d x^{\prime},
$$

где $X_{0}$ - проекция множества $X$ на $I^{n-1}$, а $\nabla f-$ градиент функции $f . Э_{\text {тот }}$ градиент ограничен, и мы видим, что $m\left(X_{0}\right) \leqslant m(X) \leqslant C_{3} m\left(X_{0}\right)$, где $C_{3}-$ постоянная, так что меры $m(X)$ и $m\left(X_{0}\right)$ эквивалентны.

С другой стороны, рассмотрим малые $n$-мерные кубы $V^{\alpha}$, имеющие общие точки с $\mathfrak{S}$ и лежашие в «вертикальном цилиндре», основание которого - малый $(n-1)$-мерный куб $V^{\alpha^{\prime}}$, лежащий в $I^{n-1}$. Поскольку функция $f$ липшицева, число этих $V^{\alpha}$ ограничено некоторой постоянной $C_{4}$. Мы видим, что $\mu\left(X_{0}\right) \leqslant$ $\mu(X) \leqslant C_{4} \mu\left(X_{0}\right)$, так что величины $\mu\left(X_{0}\right)$ и $\mu(X)$ также эквивалентны.

Как следствие получаем, что эквивалентны величины $\mu(X)$ и $m(X)$.

3) Теперь пусть $X$ лежит на поверхности $\mathfrak{S}$, которая является образом рассмотренной в п. 2) поверхности при некотором повороте, $\mathfrak{S} \subset I^{n}$. Заметим, что если $V$ - произвольно ориентированный $n$-мерный куб со стороной $1 / N$, то 
число малых кубов $V^{\alpha}$ разбиения $I^{n}$ с той же стороной, имеющих обшие точки с $V$, ограничено постоянной $C_{5}$, не зависящей от $N$ и ориентации куба $V$. Отсюда видно, что при произвольном повороте поверхности $\mathfrak{S}$ из п. 2) (или, что то же, при повороте системы координат в $\mathbb{R}^{n}$ ) величина $\mu(X)$ не может увеличиться более чем в $C_{5}$ раз и не может уменьшиться более чем в $C_{5}$ раз. Мера $m(X)$, конечно, не изменится. Значит, эквивалентность $\mu(X)$ и $m(X)$ сохраняется.

4) Наконец, пусть $X$ - любое замкнутое подмножество на замкнутой липшицевой поверхности $S$, лежащей в $I^{n}$. Предположим, что мы можем представить $X$ в виде объединения замкнутых подмножеств $X_{1}, \ldots, X_{K}$, причем $K$ не зависит от $X, m\left(X_{i} \cap X_{j}\right)=0(i \neq j)$ и $C^{\prime} \leqslant \mu\left(X_{j}\right) / m\left(X_{j}\right) \leqslant C^{\prime \prime}$, где $C^{\prime}$ и $C^{\prime \prime}$ - положительные постоянные, не зависящие от множества $X$ и его разбиения на части $X_{j}$. Тогда

$$
\begin{gathered}
\mu(X) \leqslant \mu\left(X_{1}\right)+\cdots+\mu\left(X_{K}\right) \leqslant K \mu(X), \\
m(X)=m\left(X_{1}\right)+\cdots+m\left(X_{K}\right), \\
C^{\prime} \leqslant \frac{\mu\left(X_{1}\right)+\cdots+\mu\left(X_{K}\right)}{m\left(X_{1}\right)+\cdots+m\left(X_{K}\right)} \leqslant C^{\prime \prime},
\end{gathered}
$$

и мы получаем, что $C^{\prime} / K \leqslant \mu(X) / m(X) \leqslant C^{\prime \prime}$, т. е. приходим к цели.

Остается убедиться в возможности разбиения $X$ на части $X_{j}$ с нужными свойствами. Поверхность $S$ допускает конечное покрытие системой связных открытых подмножеств $O_{j}(j=1, \ldots, K)$, замыкание каждого из которых лежит на графике липшицевой функции, с $m\left(\partial O_{j}\right)=0$ для всех $j$. Положим

$$
U_{1}=O_{1}, U_{2}=O_{2} \backslash U_{1}, \ldots, U_{K}=O_{K} \backslash \bigcup_{1}^{K-1} U_{j} .
$$

Эти множества измеримы и попарно не пересекаются, их объединение есть $S$, замыкание $\bar{U}_{j}$ при каждом $j$ лежит на графике липшицевой функции, и $m\left(\bar{U}_{j} \cap \bar{U}_{k}\right)=0$ при $j \neq k$. Остается положить $X_{j}=\overline{X \cap U_{j}}$.

Отметим, что разбиение поверхности $S$ на части $U_{j}$ будет еще раз использовано в доказательстве теоремы 3.2 .

ЗАмечАния. Отметим, что предположение о замкнутости $X$ в лемме существенно. Например, если $X$ - счетное всюду плотное множество на $S$, то $m(X)=0$ и $\mu(X)=\mu(S)=m(S)$. Но незамкнутые $X$ нам не понадобятся. Отметим также, что величина $\mu(X)$, вообе говоря, не инвариантна относительно поворотов: в этом легко убедиться на примере отрезка $X=\mathfrak{S}$ при $n=2$.

Положим $m_{\varphi}=m(\operatorname{supp} \varphi)$,

$$
\begin{gathered}
\gamma=1+q /(n-1), \\
d(\varphi, \psi)=\min \left(m_{\varphi}^{\gamma}, m_{\psi}^{\gamma}\right), \\
d_{1}(\varphi, \psi)=\min \left(\mu_{\varphi}^{\gamma}, \mu_{\psi}^{\gamma}\right) .
\end{gathered}
$$

В силу леммы 2.2 величины $d(\varphi, \psi)$ и $d_{1}(\varphi, \psi)$ эквивалентны. Ниже удобнее пользоваться величиной $d_{1}(\varphi, \psi)$, но в окончательном результате мы заменим 
ее величиной $d(\varphi, \psi)$, инвариантной относительно поворотов системы координат в $\mathbb{R}^{n}$.

ПРЕДЛОЖЕНИЕ 2.3. Для оператора (1.2) с $K \in \mathcal{K}^{q}$ справедлива оченка

$$
\varlimsup_{j \rightarrow \infty} s_{j}(A) j^{\gamma} \leqslant C d_{1}(\varphi, \psi),
$$

әде постоянная $C$ не зависит от $\varphi$ и $\psi$.

ДокАЗАтЕльство. Оценивая размерность оператора (2.3), можно принять, что $K_{N}(x, y) \equiv 0$ при $x \in V^{\alpha}$, если $\operatorname{supp} \varphi$ не пересекается с $V^{\alpha}$. Поэтому

$$
\operatorname{dim} A_{N} \leqslant C(n, r) \nu_{\varphi}(N) \leqslant 2 C(n, r) \mu_{\varphi} N^{n-1}
$$

при достаточно больших $N$, где $C(n, r)$ - размерность пространства многочленов порядка не выше $r$ от $n$ переменных. Кроме того, так как

$$
\left\|A-A_{N}\right\|=\left\|\varphi\left(K-K_{N}\right) \psi\right\| \leqslant\left\|K-K_{N}\right\|,
$$

то в силу (2.6) имеем $\left\|A-A_{N}\right\| \leqslant C_{1} N^{-q-n+1}$, где $C_{1}$ не зависит от $\varphi$ и $\psi$. Применяя лемму 2.1, получаем оценку

$$
\varlimsup_{j \rightarrow \infty} s_{j}(A) j^{\gamma} \leqslant C_{1}\left(2 C(n, r) \mu_{\varphi}\right)^{\gamma} .
$$

Аналогичная оценка с $\mu_{\psi}$ вместо $\mu_{\varphi}$ получается, если вместо $A$ рассмотреть имеющий те же $s$-числа сопряженный к $A$ оператор

$$
A^{*} u(x)=\int_{S} \overline{\psi(x) K(y, x) \varphi(y)} u(y) d S_{y} \quad(x \in S) .
$$

2.1b. Для оценки нормы $\left\|A-A_{N}\right\|$ нам понадобится

ЛЕмма 2.4. Пусть ядро $w$ оператора (2.4) удовлетворяет неравенству

$$
|w(x, y)| \leqslant h(|x-y|)
$$

при $x \neq y$, где функчия $h(t)$ такова, что при некотором $\lambda \geqslant 0$ функция $t^{-\lambda} h(t)$ не возрастает на $(0, \sqrt{n})$. Тогда

$$
\|w\| \leqslant C \int_{0}^{\sqrt{n}} t^{n-2} h(t) d t
$$

әде постоянная $C$ зависит только от поверхности $S$.

ДокАЗАТЕЛЬСтво. В силу известного неравенства

$$
\|w\|^{2}=\|W\|^{2} \leqslant \sup _{x} \int_{S}|w(x, y)| d S_{y} \cdot \sup _{y} \int_{S}|w(x, y)| d S_{x}
$$

имеем

$$
\|w\| \leqslant \sup _{x} \int_{S} h(|x-y|) d S_{y} .
$$

Достаточно рассмотреть поверхность $\mathfrak{S}$, заданную в форме (1.1). Ясно, что в этом случае $\left|x^{\prime}-y^{\prime}\right| \leqslant|x-y| \leqslant \sqrt{1+L^{2}}\left|x^{\prime}-y^{\prime}\right|$. Учитывая монотонность 
$t^{-\lambda} h(t)$ и неравенство $\operatorname{diam}(\mathfrak{S}) \leqslant \sqrt{n}$, получаем из $(2.17)$, полагая $g\left(y^{\prime}\right)=$ $\sqrt{1+\left|\nabla f\left(y^{\prime}\right)\right|^{2}}$ :

$$
\begin{aligned}
\|w\| & \leqslant \max _{x} \int_{\mathfrak{S}}|x-y|^{-\lambda} h(|x-y|)|x-y|^{\lambda} d S_{y} \\
& \leqslant \max _{x} \int_{\Omega}\left|x^{\prime}-y^{\prime}\right|^{-\lambda} h\left(\left|x^{\prime}-y^{\prime}\right|\right)\left(1+L^{2}\right)^{\lambda / 2}\left|x^{\prime}-y^{\prime}\right|^{\lambda} g\left(y^{\prime}\right) d y^{\prime} \\
& \leqslant C_{1} \int_{\left|y^{\prime}\right| \leqslant \sqrt{n}} h\left(\left|y^{\prime}\right|\right) d y^{\prime}=C_{2} \int_{0}^{\sqrt{n}} t^{n-2} h(t) d t .
\end{aligned}
$$

2.2. Здесь мы рассмотрим некоторые нужные нам подклассы класса $\mathcal{K}^{q}$ и покажем, в частности, что в него входят ядра всех полиоднородных ПДО порядка $-q-n+1$ в $\mathbb{R}^{n}$ или $\mathcal{O}$.

2.2а. Рассмотрим ядра $K(x, y)=L(x, y, x-y)$, где $L(x, y, z)-$ функция из $C^{l}\left(\overline{\mathcal{O}} \times \overline{\mathcal{O}} \times\left(\mathbb{R}^{n} \backslash 0\right)\right)$, положительно однородная степени $q \in \mathbb{R}$ по переменной $z$, где $q>-n+1$ и $l \in \mathbb{N}$. Класс таких ядер мы обозначим через $\mathcal{H}^{q, l}$.

ПреДЛОЖЕНИЕ 2.5. $\mathcal{H}^{q, l} \subset \mathcal{K}^{p}$, где

$$
p= \begin{cases}q, & q<l-n+1, \\ q-\varepsilon, & q=l-n+1, \\ l-n+1, & q>l-n+1,\end{cases}
$$

$u \varepsilon-$ сколь угодно малое положительное число.

ДокаЗАТЕЛЬство. Будем обозначать через $\hat{x}$ центр куба $V^{\alpha} \ni x$, а сам этот куб $V^{\alpha}$ - через $V_{x}$. Так как $|x-\hat{x}| \leqslant \sqrt{n} /(2 N)$, то

$$
\begin{aligned}
|x-y| \leqslant \sqrt{n} / N & \Longrightarrow|\hat{x}-\hat{y}| \leqslant 2 \sqrt{n} / N, \\
|x-y|>3 \sqrt{n} / N & \Longrightarrow|\hat{x}-\hat{y}|>2 \sqrt{n} / N
\end{aligned}
$$

и, как нетрудно проверить,

$$
|\hat{x}-\hat{y}|>2 \sqrt{n} / N \Longrightarrow 1 / 2 \leqslant|x-y| /|\hat{x}-\hat{y}| \leqslant 3 / 2 .
$$

Для ядра $K(x, y)=L(x, y, x-y)$ положим

$$
K_{N}(x, y)= \begin{cases}0, & |\hat{x}-\hat{y}| \leqslant 2 \sqrt{n} / N \\ T_{l-1}(x, y), & |\hat{x}-\hat{y}|>2 \sqrt{n} / N\end{cases}
$$

где $T_{l-1}(x, y)$ - многочлен Тейлора порядка $l-1$ по переменной $x$ с центром в точке $\hat{x}$ для функции $F(x)=L(x, y, x-y)$.

Не ограничивая общности, примем, что $K$ - вещественнозначная функция. В силу формулы Тейлора с остаточным членом в форме Лагранжа,

$$
\left|K(x, y)-T_{l-1}(x, y)\right| \leqslant \sum_{|\beta|=l} C_{\beta}\left|\partial_{x}^{\beta} L(x, y, x-y)\right|_{x=\tilde{x}}(x-\hat{x})^{\beta} \mid,
$$

где $\tilde{x} \in V_{x}$; поэтому при $|\hat{x}-\hat{y}|>2 \sqrt{n} / N$, используя $(2.20)$, имеем

$$
\left|K(x, y)-K_{N}(x, y)\right| \leqslant C_{1} \sum_{j=0}^{l}|\tilde{x}-y|^{q-j} N^{-l} \leqslant C_{2}|x-y|^{q-l} N^{-l} .
$$


При $|\hat{x}-\hat{y}| \leqslant 2 \sqrt{n} / N$, в силу однородности $L(x, y, z)$ по $z$,

$$
\left|K(x, y)-K_{N}(x, y)\right|=|K(x, y)| \leqslant C_{3}|x-y|^{q} .
$$

Отсюда, используя импликации (2.19) и соотношение

$$
t^{q-l} N^{-l} \asymp t^{q} \quad \text { при } \quad t \asymp 1 / N
$$

(где запись $f \asymp g$ означает, что $f$ и $g$ эквивалентны, т. е. дробь $f / g$ заключена между положительными постоянными), получаем для погрешности аппроксимации оценку

$$
\left|K(x, y)-K_{N}(x, y)\right| \leqslant h(|x-y|)
$$

с функцией

$$
h(t)= \begin{cases}C_{4} t^{q-l} N^{-l}, & t>1 / N, \\ C_{4} t^{q}, & t \leqslant 1 / N,\end{cases}
$$

где $C_{4}$ - достаточно большая постоянная, не зависящая от $N$.

Применяя лемму 2.4, получаем оценку

$$
\left\|K-K_{N}\right\| \leqslant \begin{cases}C_{5} N^{-q-n+1}+C_{6} N^{-l}, & l \neq q+n-1, \\ C_{6} N^{-l} \ln N, & l=q+n-1,\end{cases}
$$

из которой вытекает нужный результат, так как $\overline{K(y, x)}$ входит в класс $\mathcal{H}^{q, l}$ вместе с $K(x, y)$.

2.2b. Рассмотрим ядра $K(x, y)=P(x, y, x-y) \ln |x-y|$, где $P(x, y, z)-$ однородный степени $q \in \mathbb{Z}_{+}$многочлен по переменной $z$ с коэффициентами из $C^{l}(\overline{\mathcal{O}} \times \overline{\mathcal{O}}), l \in \mathbb{N}$. Этот класс ядер обозначим через $\mathcal{H}_{\ln }^{q, l}$.

ПРЕДЛОЖЕНИЕ 2.6. $\mathcal{H}_{\mathrm{ln}}^{q, l} \subset \mathcal{K}^{p}$, әде

$$
p= \begin{cases}q, & q<l-n+1, \\ l-n+1-\varepsilon, & q \geqslant l-n+1,\end{cases}
$$

$u \varepsilon-$ сколь угодно малое положительное число.

ДоКАЗАТЕЛЬСТВО. Положим

$$
K_{N}(x, y)= \begin{cases}-Q_{l-1}(x, y) \ln N, & |\hat{x}-\hat{y}| \leqslant 2 \sqrt{n} / N \\ Q_{l-1}(x, y) T_{l-1}(x, y), & |\hat{x}-\hat{y}|>2 \sqrt{n} / N\end{cases}
$$

где $T_{l-1}(x, y)$ - многочлен Тейлора порядка $l-1$ по переменной $x$ с центром в точке $\hat{x}$ для функции $\ln |x-y|$, а $Q_{l-1}(x, y)$ - многочлен Тейлора порядка $l-1$ по переменной $x$ с центром в точке $\hat{x}$ для функции $P(x, y, x-y)$.

Не ограничивая общности, примем, что $P$ имеет вещественные значения. В силу формулы Тейлора с остаточным членом в форме Лагранжа,

$$
\left|P(x, y, x-y)-Q_{l-1}(x, y)\right| \leqslant \sum_{|\beta|=l} C_{\beta}\left|\partial_{x}^{\beta} P(x, y, x-y)\right|_{x=\hat{x}}(x-\hat{x})^{\beta} \mid,
$$

где $\tilde{x} \in V_{x}$. Отсюда получаем, что

$$
\left|P(x, y, x-y)-Q_{l-1}(x, y)\right| \leqslant C_{1} N^{-l} .
$$


Поэтому при $|\hat{x}-\hat{y}| \leqslant 2 \sqrt{n} / N$ имеем (используя на последнем шаге $(2.19 \mathrm{~b})$ )

$$
\begin{gathered}
\left|K(x, y)-K_{N}(x, y)\right|=|P(x, y, x-y) \ln | x-y\left|+Q_{l-1}(x, y) \ln N\right| \\
\leqslant\left|Q_{l-1}(x, y)-P(x, y, x-y)\right| \ln N+|P(x, y, x-y)(\ln |x-y|+\ln N)| \\
\leqslant C_{1} N^{-l} \ln N+|P(x, y, x-y) \ln (|x-y| N)| \\
\leqslant C_{1} N^{-l} \ln N+C_{2}|x-y|^{q}\left(\ln \frac{3 \sqrt{n}}{|x-y| N}+1\right)
\end{gathered}
$$

Снова в силу формулы Тейлора с остаточным членом в форме Лагранжа,

$$
|\ln | x-y\left|-T_{l-1}(x, y)\right| \leqslant \sum_{|\beta|=l} C_{\beta}\left|\partial_{x}^{\beta} \ln \right| x-y||_{x=\tilde{x}}(x-\hat{x})^{\beta} \mid,
$$

где $\tilde{x} \in V_{x}$. Поэтому при $|\hat{x}-\hat{y}|>2 \sqrt{n} / N$, используя $(2.20)$, имеем

$$
|\ln | x-y\left|-T_{l-1}(x, y)\right| \leqslant C_{3}|\tilde{x}-y|^{-l} N^{-l} \leqslant C_{4}|x-y|^{-l} N^{-l},
$$

откуда, в частности, следует (с учетом (2.19a)), что

$$
\left|T_{l-1}(x, y)\right| \leqslant C_{4}|x-y|^{-l} N^{-l}+|\ln | x-y|| \leqslant C_{5}+\ln N .
$$

Используя оценки $(2.26),(2.29),(2.28)$ и однородность $P$, получаем следующую оценку при $|\hat{x}-\hat{y}|>2 \sqrt{n} / N$ :

$$
\begin{aligned}
\left|K(x, y)-K_{N}(x, y)\right| & =|P(x, y, x-y) \ln | x-y\left|-Q_{l-1}(x, y) T_{l-1}(x, y)\right| \\
\leqslant & \left|\left(P(x, y, x-y)-Q_{l-1}(x, y)\right) T_{l-1}(x, y)\right| \\
& \quad+\left|P(x, y, x-y)\left(\ln |x-y|-T_{l-1}(x, y)\right)\right| \\
\leqslant & C_{1} N^{-l}\left(C_{5}+\ln N\right)+C_{6}|x-y|^{q-l} N^{-l} .
\end{aligned}
$$

Используя импликации (2.19) и соотношение (2.22), получаем для погрешности аппроксимации из неравенств (2.30) и (2.27) оценку (2.23) с функцией

$$
h(t)=C_{7} N^{-l} \ln N+ \begin{cases}C_{7} t^{q-l} N^{-l}, & t>1 / N, \\ C_{7} t^{q}(1-\ln (t N)), & t \leqslant 1 / N,\end{cases}
$$

где $C_{7}$ - достаточно большая постоянная, не зависящая от $N$.

Применяя лемму 2.4, получаем оценку

$$
\left\|A-A_{N}\right\| \leqslant C_{8} N^{-l} \ln N+C_{8} N^{-q-n+1},
$$

из которой вытекает нужный результат, так как $\overline{K(y, x)}$ содержится в классе $\mathcal{H}_{\text {ln }}^{q, l}$ вместе с $K(x, y)$.

2.2c. ПРеДЛОЖЕНИЕ 2.7. Пусть $K(x, y) \in C^{l}(\overline{\mathcal{O}} \times \overline{\mathcal{O}}), l \in \mathbb{N}$. Тогда $K(x, y)$ $\in \mathcal{K}^{q}$, где $q=l-n+1$.

ДокАЗАтЕльство. В качестве аппроксимации $K_{N}(x, y)$ возьмем многочлен Тейлора $T_{l-1}(x, y)$ порядка $l-1$ по переменной $x$ для функции $K(x, y)$ с центром в точке $\hat{x}$. Предположив функцию $K$ вещественнозначной, при помощи 
формулы Тейлора с остаточным членом в форме Лагранжа получаем

$$
\left|K(x, y)-T_{l-1}(x, y)\right| \leqslant C_{1} \sum_{|\beta|=l} \max \left|\partial_{x}^{\beta} K(x, y)\right| \cdot\left|(x-\hat{x})^{\beta}\right|
$$

(где $C_{1}$ не зависит от $K$ ); поэтому $\left|K(x, y)-K_{N}(x, y)\right| \leqslant C_{2} N^{-l}$. Применяя лемму 2.4 , получаем

$$
\left\|A-A_{N}\right\| \leqslant C_{2} N^{-l} \int_{0}^{\sqrt{n}} t^{n-2} d t=C_{3} N^{-l} .
$$

2.2d. Пусть $q>-n+1$ и $l=[q]+n$. Рассмотрим ядро $K(x, y)$ вида

$$
K(x, y)=\sum_{j=0}^{k} K_{q_{j}}(x, y)+L(x, y)
$$

где $q=q_{0}<q_{1}<\cdots<q_{k} ; K_{q_{j}}(x, y) \in \mathcal{H}^{q_{j}, l}$, если $q_{j} \notin \mathbb{Z}_{+}$, и $K_{q_{j}}(x, y) \in$ $\mathcal{H}^{q_{j}, l}+\mathcal{H}_{\mathrm{ln}}^{q_{j}, l}$ (т.е. $K_{q_{j}}$ — сумма ядер из этих двух классов), если $q_{j} \in \mathbb{Z}_{+}$; $L(x, y) \in C^{l}(\overline{\mathcal{O}} \times \overline{\mathcal{O}})$. Класс ядер вида $(2.31)$ мы будем обозначать через $\mathcal{P}^{q}$.

В этот класс входят ядра всех полиоднородных ПДО порядка $m-1=-q-n$ в $\mathbb{R}^{n}$ (см. [1] или [2]). Из предложений 2.5-2.7 вытекает

ПРЕДЛОЖЕНИЕ 2.8. $\mathcal{P}^{q} \subset \mathcal{K}^{q}$.

Сформулируем основной результат этого параграфа, вытекающий из предложений 2.3 и 2.7, в виде следующей теоремы. Напомним, что величина $d(\varphi, \psi)$ определена в (2.11).

Теорема 2.9. Пусть $K(x, y) \in \mathcal{P}^{q}$. Тогда s-uисла оператора (1.2) удовлетворяют неравенству

$$
\varlimsup_{j \rightarrow \infty} s_{j}(A) j^{1+q /(n-1)} \leqslant C d(\varphi, \psi) .
$$

Оценка (2.32) совпадает с (1.3). Этот результат без труда переносится на матричные операторы.

ЗАмЕчАниЕ 2.10. Если $S$ - область в $\mathbb{R}^{n-1}$ или $(n-1)$-мерная бесконечно гладкая замкнутая поверхность, то оценка вида (2.32) справедлива для операторов с ядрами, заданными только на $S \times S$. В этом случае аппроксимации ядер строятся теми же способами в пределах $S \times S$. Мы не будем на этом останавливаться. Сp. [3, 4].

\section{§3. Асимптотика собственных значений самосопряженных ПДО отрицательного порядка на почти гладкой поверхности}

3.1. В этом параграфе мы наложим более жесткие условия на оператор $A$ и поверхность $S$. Поверхность $S$ будем считать почти гладкой в следующем смысле.

ОПРЕДЕЛЕНИЕ 3.1. Замкнутую липшицеву $(n-1)$-мерную поверхность $S$ в $\mathbb{R}^{n}$ назовем почти гладкой, если вне некоторого замкнутого подмножества 
$S_{0}$ с $m\left(S_{0}\right)=\mu\left(S_{0}\right)=0$ она имеет структуру открытого бесконечно гладкого $(n-1)$-мерного подмногообразия в $\mathbb{R}^{n}$.

Это условие можно понимать следующим образом. В любом локальном представлении (1.1) поверхности $S$ в виде графика липшицевой функции эта функция принадлежит $C^{\infty}$ вне некоторого подмножества в $\Omega$, замыкание которого имеет нулевую $(n-1)$-мерную меру. Отметим, что множество $S_{1}=S \backslash S_{0}$ может быть несвязным. Множество $S_{0}$ назовем множеством особенностей поверхности $S$.

Как мы уже отмечали, поверхности конуса и цилиндра с круговыми основаниями и многогранника в $\mathbb{R}^{3}$ - примеры почти гладких поверхностей; в первом случае $S_{0}$ состоит из вершины и всех точек края основания, в третьем - из всех точек ребер многогранника. Нетрудно построить почти гладкий «многогранник» в $\mathbb{R}^{3}$ со счетным множеством граней.

Оператор $\mathcal{A}$ в открытом множестве $\mathcal{O} \supset S$ мы теперь будем считать полиоднородным матричным ПДО порядка $m-1, m<0$; в частности, он будет иметь ядро $K(x, y)$, бесконечно гладкое при $x \neq y$. Пусть интегральный оператор $A_{0}$ на $S$ имеет ядро, являюшееся сужением ядра $K(x, y)$ на $S \times S$. В $O^{\prime}\left(x_{0}\right)$ для любой точки $x_{0} \in S_{1}$ это полиоднородный ПДО (например, в том смысле, что если $\alpha, \beta$ - функции из $C_{0}^{\infty}\left(O^{\prime}\left(x_{0}\right)\right)$, то $\alpha A_{0}(\beta \cdot)$ - полиоднородный ПДО в $\left.O^{\prime}\left(x_{0}\right)\right)$ порядка $m<0$. Обозначим через $a_{0}\left(x^{\prime}, \xi^{\prime}\right)$ главный символ этого ПДО $A_{0}$; ниже в предложении 3.5 мы выразим его через главный символ ПДО $\mathcal{A}$.

Введем функции распределения собственных чисел компактного оператора $T$ в гильбертовом пространстве $H$. Через $N_{+}(t, T)$ и $N_{-}(t, T)$, где $t>0$, обозначим число собственных значений этого оператора, больших $t$ и меньших $-t$ соответственно; при этом собственные значения подсчитываются с учетом их кратностей. Аналогичным образом определим величины $n_{+}(t, a)$ и $n_{-}(t, a)$ для квадратной числовой матрищы $a$.

Теперь мы можем сформулировать основную теорему в настоящем параграфе.

ТЕОРема 3.2. Пусть $\varphi$ - ограниченная измеримая функиия на почти гладкой липшичевой поверхности $S$, и пусть $A=\varphi A_{0}(\bar{\varphi} \cdot)$ - самосопряженный оператор в $L^{2}(S)$. Тогда

$$
N_{ \pm}(t, A)=c_{ \pm} t^{\frac{n-1}{m}}+o\left(t^{\frac{n-1}{m}}\right) \quad n p u t \rightarrow 0
$$

$2 \partial e$

$$
c_{ \pm}=\frac{1}{(2 \pi)^{n-1}} \iint_{T^{*} S_{1}} n_{ \pm}\left(1,\left|\varphi\left(x^{\prime}\right)\right|^{2} a_{0}\left(x^{\prime}, \xi^{\prime}\right)\right) d x^{\prime} d \xi^{\prime}
$$

3.2. В доказательстве будут использованы два следующих результата, принадлежащих М. Ш. Бирману и М. 3. Соломяку (см. [4] и [14]).

ПРЕДЛОЖЕНИЕ 3.3. Пусть $A_{0}$ - полиоднородный ПДО порядка $m<0$ в ограниченной области $O^{\prime}$ пространства $\mathbb{R}^{n-1}$, и пусть $\varphi\left(x^{\prime}\right)$ - ограниченная измеримая функиия с носителем, лежащим в $O^{\prime}$. Предположим, что $A=\varphi A_{0}(\bar{\varphi} \cdot)$ - самосопряженный оператор в $L^{2}\left(O^{\prime}\right)$. Тогда справедливь формульи (3.1)-(3.2) с заменой $S_{1}$ на $O^{\prime}$.

ПРЕДЛОЖЕНИЕ 3.4. Пусть $T$ - компактный самосопряженный оператор в аильбертовом пространстве $H$, и пусть при некотором $\sigma>0$ для 
любого $\varepsilon>0$ оператор $T$ представим в виде суммь $T_{\varepsilon}^{\prime}+T_{\varepsilon}^{\prime \prime}$ компактных самосопряженных операторов, таких, что существуют конечнье предель

$$
\lim _{t \rightarrow 0} N_{ \pm}\left(t, T_{\varepsilon}^{\prime}\right) t^{\sigma}=c_{ \pm}\left(T_{\varepsilon}^{\prime}\right)
$$

$u$

$$
\varlimsup_{j \rightarrow \infty} s_{j}\left(T_{\varepsilon}^{\prime \prime}\right) j^{1 / \sigma} \leqslant \varepsilon .
$$

Тогда при $\varepsilon \rightarrow 0$ величинь $c_{ \pm}\left(T_{\varepsilon}^{\prime}\right)$ стремятся $\kappa$ конечньм пределам $c_{ \pm} u$

$$
\lim _{t \rightarrow 0} N_{ \pm}(t, T) t^{\sigma}=c_{ \pm}
$$

ДОКАЗАТЕЛЬСТВо ТЕОРЕмЫ 3.2. Воспользуемся разбиением поверхности $S$ на части (2.9). Положим

$$
S_{0}^{\prime}=S_{0} \cup \partial U_{1} \cup \cdots \cup \partial U_{k} .
$$

Это замкнутое подмножество на $S$ нулевой меры. Обозначим через $S_{\delta}^{\prime}$ его открытую $\delta$-окрестность. Зафиксируем $\varepsilon>0$ и найдем такое $\delta>0$, что $m\left(S_{\delta}^{\prime}\right)<\varepsilon$. Зафиксируем $\delta$ и положим

$$
S^{\prime}=S_{\delta}^{\prime}, \quad S^{\prime \prime}=S \backslash S^{\prime} \text { и } D_{j}=U_{j} \backslash S^{\prime}(j=1, \ldots, K) .
$$

Множества $D_{j}$ замкнуты, попарно не пересекаются и вместе составляют $S^{\prime \prime}$.

Обозначим через $\varphi_{k}$ индикатор множества $D_{k}(k=1, \ldots, p)$ и через $\varphi_{0}$ индикатор множества $S^{\prime}$. Мы имеем

$$
A=A^{\prime}+A^{\prime \prime}, \quad \text { где } \quad A^{\prime}=\sum_{1}^{p} \varphi_{k} A\left(\varphi_{k} \cdot\right) \quad \text { и } \quad A^{\prime \prime}=\sum_{l, k}^{\prime} \varphi_{k} A\left(\varphi_{l} \cdot\right) ;
$$

в последней сумме в каждом слагаемом либо $k \geqslant 1, l \geqslant 1$ и $k \neq l$, либо $l$ и/или $k$ равно 0 . Так как носители функций $\varphi_{k}(k \geqslant 1)$ не пересекаются, то

$$
N_{ \pm}\left(t, A^{\prime}\right)=\sum_{1}^{p} N_{ \pm}\left(t, \varphi_{k} A\left(\varphi_{k} \cdot\right)\right)
$$

где к каждому слагаемому применимо предложение 3.3. Значит, эта сумма имеет вид $(3.1)$, где $c_{ \pm}$- суммы соответствующих интегралов по $T^{*} D_{k}$. Очевидно, что эти суммы стремятся к (3.2) при $\varepsilon \rightarrow 0$.

Теперь оценим $s$-числа оператора $A^{\prime \prime}$. В силу теоремы 2.9 при больших $j$

$$
s_{j}\left(\varphi_{0} A\left(\sum_{0}^{p} \varphi_{k} \cdot\right)\right) \leqslant C \varepsilon j^{\frac{m}{n-1}} \quad \text { и } \quad s_{j}\left(\sum_{1}^{p} \varphi_{k} A\left(\varphi_{0} \cdot\right)\right) \leqslant C \varepsilon j^{\frac{m}{n-1}},
$$

где $C$ - постоянная, не зависящая от $\varepsilon$ и $j$. В остальных слагаемых носители функций $\varphi_{k}$ и $\varphi_{l}$ не пересекаются, так что это произведения ограниченных операторов и интегрального оператора с бесконечно гладким ядром. Поэтому $s$-числа этих слагаемых быстро убывают, и то же верно для их суммы, так как для любых двух компактных операторов $T_{1}$ и $T_{2}$

$$
s_{j+k-1}\left(T_{1}+T_{2}\right) \leqslant s_{j}\left(T_{1}\right)+s_{k}\left(T_{2}\right)
$$


(см. [12, гл. II]). Мы заключаем, что $\varlimsup s_{j}\left(A^{\prime \prime}\right) j^{-m /(n-1)} \leqslant C^{\prime} \varepsilon$, где постоянная $C^{\prime}$ не зависит от $\varepsilon$. Остается применить предложение 3.4 .

ЗАмЕчАния. Как частный случай теорема 3.2 содержит аналог предложения 3.3 Бирмана-Соломяка для ПДО на гладком замкнутом многообразии. В этом случае теорема 3.2 легко выводится из результатов в [4] и без предположения, что $A_{0}$ получается из $A$ сужением ядра на $S \times S$. Мы тоже могли бы в этом случае обойтись без этого предположения с учетом замечания 2.10 .

В [4] ядра и символы предполагались имеющими некоторую конечную гладкость; поэтому и теорема 3.2 допускает ослабление предположений о гладкости.

3.4. Укажем формулу для вычисления главного символа ПДО $A_{0}$ через главный символ ПДО $\mathcal{A}$. Рассмотрим окрестность $O\left(x_{0}\right)$ из определения 3.1 и перейдем в ней к координатам $\left(x^{\prime}, y_{n}\right)$, где $y_{n}=x_{n}-f\left(x^{\prime}\right)$, но для единообразия переобозначим $y_{n}$ заново через $x_{n}$. Пусть $\alpha(x, \xi)$ - главный символ ПДО $\mathcal{A}$ в этих координатах.

ПрЕДЛОЖЕНИЕ 3.5. При $\xi^{\prime} \neq 0$

$$
a_{0}\left(x^{\prime}, \xi^{\prime}\right)=\frac{1}{2 \pi} \int \alpha_{0}\left(x^{\prime}, 0, \xi^{\prime}, \xi_{n}\right) d \xi_{n} .
$$

Это сходящийся интеграл, так как

$$
\left|\alpha_{0}(x, \xi)\right| \leqslant C_{1}|\xi|^{m-1} .
$$

Для доказательства предложения 3.5 можно умножить $\mathcal{A}$ слева и справа на финитную бесконечно гладкую функцию с носителем в $O\left(x_{0}\right)$, равную 1 вблизи рассматриваемой точки. Это не отражается на главном символе. Продолжая теперь ядро нулем вне $O\left(x_{0}\right) \times O\left(x_{0}\right)$, можно принять, что $\mathcal{A}-\Pi$ ПО в $\mathbb{R}^{n}$. Перепишем его ядро $K(x, y)$ в виде $K_{1}(x, x-y)$. Теперь полный символ $\alpha(x, \xi)$ ПДО $\mathcal{A}$ вычисляется по известной формуле (см., например, $[2$, п. 1.2])

$$
\alpha(x, \xi)=\int e^{i(x-y) \cdot \xi} K_{1}(x, x-y) d y=\int e^{i z \cdot \xi} K_{1}(x, z) d z .
$$

Аналогично полный символ $a\left(x^{\prime}, \xi^{\prime}\right)$ ПДО $A_{0}$, рассматриваемого как ПДО в $\mathbb{R}^{n-1}$, вычисляется по формуле

$$
a\left(x^{\prime}, \xi^{\prime}\right)=\int e^{i z^{\prime} \cdot \xi^{\prime}} K_{1}\left(x^{\prime}, 0, z^{\prime}, 0\right) d z^{\prime} .
$$

Из этих двух формул видно, что

$$
a\left(x^{\prime}, \xi^{\prime}\right)=\frac{1}{2 \pi} \int \alpha\left(x^{\prime}, 0, \xi\right) d \xi_{n} .
$$

Это аналог формулы (3.8) для локальных полных символов. Подставив сюда

$$
\alpha\left(x^{\prime}, 0, \xi\right)=\alpha_{0}\left(x^{\prime}, 0, \xi\right)+O\left(|\xi|^{m-2}\right) \quad(\xi \rightarrow \infty),
$$

получим

$$
a\left(x^{\prime}, \xi^{\prime}\right)=\frac{1}{2 \pi} \int \alpha_{0}\left(x^{\prime}, 0, \xi\right) d \xi_{n}+O\left(|\xi|^{m-1}\right) \quad(\xi \rightarrow \infty)
$$


что и дает (3.8).

3.5. Здесь мы поясним сказанное в конце введения об операторах с двойным представлением (1.5). В этом случае единственная поправка к доказательству соответствующего обобщения теоремы 3.2 состоит в следующем. Рассмотрим оператор $\varphi_{k} A \varphi_{l}$. Если нам важно, что $\mu_{\varphi_{k}}$ мало, то надо использовать второе из представлений (1.5) оператора $A$, а если важно, что мало $\mu_{\varphi_{l}}$, то первое.

\section{§4. Примеры приложений к спектральным граничным задачам. Вторая теорема об $s$-числах}

4.1. Здесь мы рассмотрим примеры, связанные с уравнением Лапласа $\Delta u=0$ в $\mathbb{R}^{3} \backslash S$, где $S$ - общая граница ограниченной области $G_{+}$и неограниченной области $G_{-}$. В качестве $K(x, y)$ возьмем стандартное фундаментальное решение $-1 /(4 \pi|x-y|)$. Пусть $\varphi(x) \equiv 1$ и $\psi(y) \equiv 1$. Сказанное о свойствах потенциалов дальше в этом пункте допускает элементарную проверку в случае бесконечно гладкой поверхности $S$ (ср. [15, §§36-37]). На случай липшицевой $S$ все переносится на основе результатов, полученных или приведенных в [9-11]. Более общие утверждения детально проверены в [16] для уравнения Гельмгольца, где обсуждаются спектральные свойства соответствующих несамосопряженных операторов.

Оператор $A$ является самосопряженным и отрицательным. Оценка (2.2) принимает вид $\left|\lambda_{j}(A)\right| \leqslant C j^{-1 / 2}$, а формула (1.4) в случае почти гладкой поверхности - вид $\lambda_{j}(A)=-c j^{-1 / 2}+o\left(j^{-1 / 2}\right)$, где $c=4 \sqrt{\pi /|S|}$ и $|S|$ - площадь поверхности $S$. Нетрудно указать граничную задачу для гармонических функций, эквивалентную уравнению $A \varphi=\lambda \varphi$. Пусть $\nu$ - единичная внешняя нормаль к $S$ (она существует на $S$ почти всюду); значками ${ }^{+}{ }_{\text {и }}{ }^{-}$условимся обозначать предельные граничные значения функций из $G_{+}$и $G_{-}$(по «регулярным» конусам $\Gamma_{ \pm}(x) \subset G_{ \pm}$с вершиной в $x \in S$ для почти всех $x \in S$; см. [9]). Задача имеет вид

$$
\Delta u=0 \quad \text { вне } S, \quad u^{+}=u^{-} \quad \text { и } \quad \lambda\left[\frac{\partial u^{-}}{\partial \nu}-\frac{\partial u^{+}}{\partial \nu}\right]=u^{ \pm} \text {на } S,
$$

при этом $u$ подчиняется условию $u(x)=O\left(r^{-1}\right)$ при $|x|=r \rightarrow \infty$. Функции $u$ и $\varphi$ связаны соотношениями

$$
\varphi=\frac{\partial u^{-}}{\partial \nu}-\frac{\partial u^{+}}{\partial \nu} \quad \text { и } \quad u(x)=-\frac{1}{4 \pi} \int_{S} \frac{\varphi(y)}{|x-y|} d y \quad(x \notin S) .
$$

При этом $\varphi \in H_{1}(S), u \in H_{3 / 2}\left(G_{+}\right) \cap C^{\infty}\left(G_{+}\right)$и $u \in H_{3 / 2, \operatorname{loc}}\left(G_{-}\right) \cap C^{\infty}\left(G_{-}\right)$, где $H_{s}$ - соболевские $L^{2}$-пространства (ср. [11]).

Остановимся еще на задаче

$$
\Delta u=0 \quad \text { в } G, \quad-u^{+}=\lambda \frac{\partial u^{+}}{\partial \nu} \text { на } S .
$$

Эта задача приводится к уравнению $T_{+} \varphi=\lambda \varphi$, где $\varphi=\partial u^{+} / \partial \nu ; u$ выражается через $\varphi$ второй формулой в $(4.2)$, и

$$
T_{+}=\left(\frac{1}{2} I-B\right)^{-1} A=A\left(\frac{1}{2} I-B^{\prime}\right)^{-1} .
$$


Через $B$ здесь обозначается прямое значение потенциала двойного слоя

$$
B \varphi(x)=-\frac{1}{4 \pi} \int_{S} \frac{\partial}{\partial \nu_{y}} \frac{1}{|x-y|} \varphi(y) d S_{y} \quad(x \in S) .
$$

На гладкой поверхности $S$ это интегральный оператор со слабой особенностью и поэтому компактный оператор в $L^{2}(S)$. На липшицевой поверхности он определен в общем случае лишь в смысле главного значения, т. е. как предел интеграла по $\{y \in S:|x-y|>\varepsilon\}$ при $\varepsilon \rightarrow 0$, в смысле сходимости почти всюду в $L^{2}(S)$ (см. ссылки в [9]), и это, вообще говоря, лишь ограниченный оператор в $L^{2}(S)$. Через $B^{\prime}$ обозначен сопряженный оператор, т. е. прямое значение нормальной производной от потенциала простого слоя; оно существует в том же смысле. Обратимость (ограниченных) операторов $\frac{1}{2} I-B$ и $\frac{1}{2} I-B^{\prime}$ в $L^{2}(S)$ и $H_{1}(S)$ доказана в [9]; там же упомянуто второе равенство в (4.4). Как и выше, $\varphi \in H_{1}(S)$ и $u \in H_{3 / 2}\left(G_{+}\right) \cap C^{\infty}\left(G_{+}\right)$. Оператор $T_{+}$- самосопряженный и отрицательный, и для него получаются такие же результаты, как для $A$, с учетом сказанного в $\S 1$ об операторах с двойным представлением (1.5); при этом постоянную $c$ в асимптотике собственных значений надо заменить на $2 c$.

4.2. Здесь мы оценим $s$-числа интегральных операторов на липшицевой поверхности с ядрами, определенными только на $S \times S$, в случае $-(n-1) / 2<$ $m<0$. Рассмотрим интегральный оператор

$$
A u(x)=\int_{S} \varphi(x) \chi(x, y)|x-y|^{q} \psi(y) u(y) d S_{y} \quad(x \in S) .
$$

Здесь $\varphi, \psi$ и $\chi$ - ограниченные измеримые функции, не превосходящие по модулю единицы, и $q$ удовлетворяет условию

$$
-(n-1)<q<-(n-1) / 2 .
$$

Положим, как и раньше, $\gamma=q /(n-1)+1$. Из $(4.7)$ следует, что $\gamma \in(0,1 / 2)$.

Теорема 4.1. При этих условиях

$$
s_{j}(A) \leqslant C \max \left(\|\varphi\|,\|\varphi\|^{\gamma}\right) \max \left(\|\psi\|,\|\psi\|^{\gamma}\right) j^{-\gamma},
$$

где $C$ - постоянная, зависящая только от $S$.

ДоказАтельство. Сначала рассмотрим случай, когда вместо $S$ мы имеем поверхность $\mathfrak{S}$, заданную в форме (1.1). Пусть $h$ - положительный параметр. Обозначим через $\theta_{h}\left(x^{\prime}\right)$ функцию на $\mathbb{R}^{n-1}$, равную 1 при $\left|x^{\prime}\right| \leqslant h$ и 0 при $\left|x^{\prime}\right|>h$. Представим оператор $A$ в виде суммы двух операторов $A_{h}^{(1)}$ и $A_{h}^{(2)}$ того же вида (4.6), но с дополнительными множителями $\theta_{h}\left(x^{\prime}-y^{\prime}\right)$ и $1-\theta_{h}\left(x^{\prime}-y^{\prime}\right)$, соответственно, под знаком интеграла. Воспользуемся тем, что $s$-числа оператора $A_{h}^{(1)}$ не превосходят его нормы (см. [12, гл. II]), а норма $\left\|A_{h}^{(1)}\right\|$ не превосходит $\sqrt{M_{1} M_{2}}$, где

$$
\begin{aligned}
& M_{1}=\max _{x} \int \theta_{h}\left(x^{\prime}-y^{\prime}\right)\left|x^{\prime}-y^{\prime}\right|^{q} \sqrt{1+\left|\nabla f\left(y^{\prime}\right)\right|^{2}} d y^{\prime}, \\
& M_{2}=\max _{y} \int \theta_{h}\left(x^{\prime}-y^{\prime}\right)\left|x^{\prime}-y^{\prime}\right|^{q} \sqrt{1+\left|\nabla f\left(x^{\prime}\right)\right|^{2}} d x^{\prime} .
\end{aligned}
$$


С учетом первого из неравенств (4.7) получаем

$$
s_{j}\left(A_{h}^{(1)}\right) \leqslant C_{1} \int_{0}^{h} r^{q+n-2} d r=C_{2} h^{q+n-1} .
$$

Теперь воспользуемся тем, что

$$
j s_{j}^{2}\left(A_{h}^{(2)}\right) \leqslant\left\|A_{h}^{(2)}\right\|^{2},
$$

где \|\|$\quad \|\left.\right|^{2}$ - квадрат нормы Гильберта-ІШмидта, т.е. интеграл от квадрата модуля ядра по $\mathfrak{S} \times \mathfrak{S}$ (см. [12, гл. III]). Имеем

$$
\begin{aligned}
\left\|A_{h}^{(2)}\right\|^{2} & \leqslant \int_{\mathfrak{S}}|\varphi(x)|^{2} d S_{x} \int_{\mathfrak{S}}\left[1-\theta_{h}\left(x^{\prime}-y^{\prime}\right)\right]^{2 q}\left|x^{\prime}-y^{\prime}\right|^{2 q} d S_{y} \\
& \leqslant C_{3}\|\varphi\|^{2} \int_{h}^{\infty} r^{2 q+n-2} d r \leqslant C_{4}\|\varphi\|^{2} h^{2 q+n-1}
\end{aligned}
$$

с учетом второго из неравенств (4.7). Аналогично

$$
\left\|A_{h}^{(2)}\right\|^{2} \leqslant C_{4}\|\psi\|^{2} h^{2 q+n-1} .
$$

Следовательно, $\left\|A_{h}^{(2)}\right\|^{2} \leqslant C_{4}\|\varphi\| \cdot\|\psi\| h^{2 q+n-1}$, и в силу (4.10)

$$
s_{j}\left(A_{h}^{(2)}\right) \leqslant C_{5} j^{-1 / 2}\|\varphi\|^{1 / 2}\|\psi\|^{1 / 2} h^{q+(n-1) / 2} .
$$

Используя неравенство (3.7), из (4.9) и (4.11) получаем

$$
s_{j}(A) \leqslant C_{6}\left(j^{-1 / 2}\|\varphi\|^{1 / 2}\|\psi\|^{1 / 2} h^{q+(n-1) / 2}+h^{q+n-1}\right) .
$$

Подставив сюда $h=\left(\|\varphi\| \cdot\|\psi\| j^{-1}\right)^{1 /(n-1)}$, мы приходим к неравенству

$$
s_{j}(A) \leqslant C_{7}\|\varphi\|^{\gamma}\|\psi\|^{\gamma} j^{-\gamma} .
$$

Перейдем к случаю замкнутой поверхности $S$. Пусть

$$
\sum_{1 \leqslant k \leqslant L} \alpha_{k}(x) \equiv 1 \quad(x \in S)
$$

- достаточно мелкое разбиение единицы на $S$ : если носители двух функций $\alpha_{i}$ и $\alpha_{k}$ имеют непустое пересечение, то объединение этих носителей лежит на графике одной липшицевой функции. Наш оператор $A$ есть сумма операторов

$$
A_{i k} u(x)=\int_{S} \alpha_{i}(x) \varphi(x) \alpha_{k}(y) \chi(x, y)|x-y|^{q} \psi(y) u(y) d S_{y} .
$$

Мы можем считать уже установленной оценку

$$
s_{j}\left(A_{i k}\right) \leqslant C_{7}\left\|\alpha_{l} \varphi\right\|^{\gamma}\left\|\alpha_{k} \psi\right\|^{\gamma} j^{-\gamma}
$$

в случаях, когда $i=k$ или $i \neq k$, но носители функций $\alpha_{i}$ и $\alpha_{k}$ пересекаются. Пусть теперь они не пересекаются. Тогда в (4.14) $|x-y|^{q}$ - ограниченная функция и $\left\|A_{j k}\right\|^{2} \leqslant C_{8}\left\|\alpha_{i} \varphi\right\|^{2}\left\|\alpha_{k} \psi\right\|^{2}$, так что

$$
s_{j}\left(A_{j k}\right)\left\|\leqslant C_{9}\right\| \alpha_{i} \varphi\|\cdot\| \alpha_{k} \psi \| j^{-1 / 2} .
$$


В (4.15) и (4.16), увеличивая константы, можно опустить множители $\alpha_{i}$ и $\alpha_{k}$. Остается снова воспользоваться неравенством (3.7), и мы получаем (4.8).

\section{ЛИТЕРАТУРА}

1. Seeley R. Refinement of the functional calculus of Calderón and Zigmund. Nederl. Akad. Wetensch. Proc. Ser. A, 68, No. 3, 521-531 (1965).

2. Агранович M. С. Эллиптические операторы на замкнутом многообразии. Итоги науки и техники, Современные проблемы математики, Фундаментальные направления, 63, 3-129, ВИНИТИ, М., 1990.

3. Бирман М. Ш., Соломяк М. З. Оценки сингулярных чисел интегральных операторов. УМН, 32, вып. 1, 17-84 (1977).

4. Бирман М. Ш., Соломяк М. З. Асимптотика спектра псевдодифференциальных операторов с анизотропно-однородными символами, I, II. Вестн. ЛГУ, №13, 1321 (1977) и №13, 5-10 (1979).

5. Костометов Г. П. Асимптотическое поведение спектра интегральных операторов с особенностью на диагонали. Матем. сб., 94 (136), №3, 444-451 (1974).

6. Ivrii V. Semiclassical Microlocal Analysis and Precise Spectral Asymptotics. Preprint 7: Chapter 10. Centre de Mathématiques, École Polytechnique, 1992.

7. Лаптев А. А. Об оценках сингулярных чисел одного класса интегральных операторов. Записки научн. семинаров ЛОМИ, 110, 95-99 (1981).

8. Nečas J. Les méthodes directes en théorie des équations elliptiques. Academia, Prague, 1967.

9. Verchota $G$. Layer potentials and boundary value problems for Laplace equation on Lipschitz domains. J. Funct. Anal., 59, 572-611 (1984).

10. Costabel $M$. Boundary integral operators on Lipschitz domains: elementary results. SIAM J. Math. Anal., 18, №3, 613-626 (1988).

11. Jerison D., Kenig C. E. The inhomogeneous Dirichlet problem in Lipschitz domains. J. Funct. Anal., 113, No. 1, 161-219 (1995).

12. Гохберг И. Ц., Крейн М. Г. Введение в теорию несамосопряженных операторов в гильбертовом пространстве. Наука, М., 1965.

13. Федерер Г. Геометрическая теория меры. Наука, М., 1987.

14. Бирман М. Ш., Соломяк М. 3. О главном члене спектральной асимптотики негладких эллиптических задач. Функц. анализ и его прилож., 4, вып. 4, 1-11 (1970).

15. Агранович M. С. Спектральные свойства задач дифракции. Добавление к книге: Войтович Н. Н., Каченеленбаум Б.З., Сивов А. Н. Обобщенный метод собственных колебаний в теории дифракции. Наука, М., 1977.

16. Агранович M. С. Спектральные задачи для уравнения Гельмгольца со спектральным параметром в граничных условиях на негладкой границе. В печати.

Московский государственный институт электроники и математики
Поступило в редакцию 25 декабря 1995 г. 